\title{
Therapeutic application of botulinum toxin
}

The neurotoxins produced by the anaerobic sporeforming Clostridium botulinum constitute the most potent group of acute toxins known. The seven serologically distinct toxins $(A-G)$ all exert similar highly specific pharmacological activity acting primarily at the neuromuscular motor synapse by blocking release of the neurotransmitter acetylcholine. This results in a flaccid muscular paralysis that is typical of the rare but often fatal disease botulism. ${ }^{1}$ Early symptoms of botulism include nausea, dry mouth and impaired vision but the disease may progress through widespread flaccid neuromuscular paralysis leading ultimately to death from respiratory depression. ${ }^{2}$ In man the most common cause of the disease is the ingestion of food containing pre-formed toxin produced by contaminating $C$. botulinum organisms. ${ }^{3}$ Infection with $C$. botulinum can give rise to other forms of botulism. Wound botulism is a rare form of the disease that results when $C$. botulinum contaminates, colonises and produces toxin in wounds in a manner analogous to the tetanus disease process. ${ }^{2,4,5}$ Infant botulism, so called because children of up to $c .35$ weeks of age only appear to be susceptible, results from colonisation of the intestinal tract by $C$. botulinum with consequent secretion of toxin. ${ }^{6,7}$

There is no known cure for the toxic paralysis of botulism. Therapeutic preparations of antitoxin are available and may form an integral part of the clinical treatment ${ }^{2}$ but by the time clinical symptoms present the toxin is irreversibly bound to the nerve terminal and the blockade of neurotransmitter release cannot be reversed. However, antitoxin therapy may improve survival and shorten the clinical course of the disease. ${ }^{2}$ Antitoxin therapy may form a useful adjunct to the antibiotic treatment used to eliminate intestinal colonisation in cases of infant botulism. ${ }^{6}$ Botulinum toxoid vaccines presently offer the only effective means of preventing the neuroparalytic effects of the botulinum toxins. Although the mortality of botulism has fallen in recent years ${ }^{2}$ the lack of any effective cure for the disease still evokes an emotive and fearful view of the toxins in both lay and scientific minds. However, the perception of these potent toxins is now changing. Because of their high potency and specificity, the toxins are now finding increasing application in the treatment of a range of clinical conditions involving uncontrolled muscle spasm, bringing relief of symptoms to many people for whom there was previously little in the way of effective treatment.

Botulinum toxins have been prepared to high purity ${ }^{8}$ and their amino-acid sequences deduced by sequencing the toxin genes. ${ }^{9}$ The toxins have similar molecular structures and mol. wts (c. $150 \mathrm{kDa})$. Although they are probably all synthesised as single polypeptide chains, these are cleaved by proteolytic enzymes to give dichain molecules comprising two asymmetric subunits-a heavy $\left(\mathrm{H}_{\mathrm{c}}\right)$ chain $(85-105 \mathrm{kDa})$ and a light $\left(\mathrm{L}_{\mathrm{c}}\right)$ chain $(50-59 \mathrm{kDa})$ linked by a disulphide bridge. The toxins are most potent in the dichain form, having specificity toxicities ranging from $2 \times 10^{7}$ to $2 \times 10^{8}$ mouse $\mathrm{LD}_{50} / \mathrm{mg}$. The molecular basis of the activity of the botulinum neurotoxins is not yet fully resolved. They do not inhibit the nerve action potential, ${ }^{7}$ nor prevent propagated potentials from entering the nerve terminal, ${ }^{10}$ nor block voltage-dependent calcium channels, ${ }^{11}$ but act by blockading exocytosis, the process whereby acetylcholine, compartmentalised within synaptic vesicles, is exported from the nerve terminal. Three stages may be involved in botulinum toxin inhibition; ${ }^{1,9}$ firstly, rapid specific and irreversible binding of toxin to acceptors on the pre-synaptic nerve surface $;^{12}$ secondly, internalisation, whereby toxin crosses the plasmalemma and enters the nerve $;{ }^{13}$ and finally, the poisoning step that disables the acetylcholine release mechanism. ${ }^{13}$ It is now clear that the toxic activity of the neurotoxins is a function of the $\mathrm{L}_{\mathrm{c}},{ }^{9,14}$ the $\mathrm{H}_{\mathrm{c}}$ being associated with the binding ${ }^{15,16}$ and internalisation ${ }^{17}$ phases. Studies involving direct injection into nerves or incorporation into permeabilised cell-culture systems have shown that the action of the $\mathrm{L}_{\mathrm{c}}$-subunit is not specifically to block acetylcholine release, as it is also capable of blocking calciummediated exocytosis of other neurotransmitter substances. The non-specificity of the intracellular toxicity indicates that the characteristic specificity of the botulinum neurotoxins is a function of their binding rather than of inhibiting activity. Various mechanisms for inhibition have been proposed, ${ }^{1,8,9}$ indeed, there is evidence that not all the botulinum toxins block neurotransmission by the same mechanism and they may affect different components of the exocytosis system. ${ }^{1}$ It has long been considered that the high specific toxicity and longevity of the inhibitory activity of the toxins indicate they are enzymic in nature. Recent exciting evidence suggests that the neurotoxins have zinc endopeptidase activity ${ }^{18.19}$ and that the toxins may act by cleaving the specific protein component(s) of synaptic vesicle membranes. ${ }^{19}$

The very characteristics that make botulinum toxin such a potent neuroparalytic agent exquisitely suit the toxin for use as a drug to induce specific muscular weakness. This novel clinical application of botulinum 
toxin type A was introduced initially as an alternative to conventional surgical intervention for the treatment of strabismus. ${ }^{20}$ The therapeutic use of botulinum toxin has extended beyond its initial application to include a variety of neurological disorders associated with involuntary spasmodic contraction of muscles, specifically focal dystonias. ${ }^{21,22}$ No specifically treatable causes of dystonia have been identified, hence only symptomatic relief can be offered to patients, but botulinum toxin therapy has proved to be remarkably effective in this context. Toxin therapy has become established as the treatment of choice for benign essential blepharospasm, in which intermittent or sustained involuntary contraction of the orbicularis oculi causes forceful closure of the eyes and is now used in preference to the surgical alternatives of muscle stripping or bilateral avulsion of the facial nerve. The treatment is not a cure, as the development of new motor end plates may restore motor function, but repeat injections usually serve to maintain effective control of the condition. ${ }^{21-23}$ Symptoms of hemifacial spasm, Meige syndrome, facial synkinesis, oromandibular dystonia and laryngeal dystonia all respond well to toxin therapy. ${ }^{22,23}$ Spasmodic torticollis is a dystonia of neck muscles that causes abnormal head movements and posture and which is frequently accompanied by cramp-like pains. Botulinum toxin therapy has proved to be beneficial for torticollis, giving temporary improvements in posture and relieving pain. ${ }^{21,22,24}$

The chemical denervation induced by botulinum toxin is increasingly being used for more complex conditions involving abnormal muscle contraction, including writer's cramp and other task-specific disorders of hand muscles. Motor dysfunction due to abnormally increased muscle tone, such as spasticity, may respond well to toxin therapy. There may also be a role for treating some forms of tremor, which is by far the most common involuntary muscular disorder. ${ }^{22,23}$

\section{References}

1. Simpson LC. The origin, structure and pharmacological activity of botulinum toxin. Pharmacol Rev 1981; 33: 155-188.

2. Tacket CO, Ragowski MA. Botulism. In: Simpson LC (ed) Botulinum neurotoxin and tetanus toxin. New York, Academic Press. 1989: 351-378

3. MacDonald KL, Cohen ML, Blake PA. The changing epidemiology of adult botulism in the United States. $A m J$ Epidemiol 1986; 124: 794-799.

4. MacDonald KL, Rutherford GW, Friedman SM et al. Botulism and botulism-like illness in chronic drug abusers. Ann Intern Med 1985; 102: 616-618.

5. Stephen J, Pietrowski RA. Bacterial toxins. Walton-on-Thames, Nelson, 1981: 1-8.

6. Arnon SS. Infant botulism. In: Boriello SP (ed) Clinical and molecular aspects of anaerobes. Petersfield, Wrightson Biomedical Publishing Ltd. 1990: 41-48.

7. Burgen ASV, Dickens F, Zatman LJ. The action of botulinum toxin on the neuromuscular junction. $J$ Physiol $1949 ; 109$ : 10-24.
There appear to be no absolute contra-indications for toxin treatment of involuntary muscular spasms. Generally, side effects are minor, self-limiting and localised and may be minimised by careful selection of dose and placement of injections. The long term effects of continued treatment are not known but there have been no reports of adverse systemic effects in patients who have received long term courses of treatment. Concerns about the possible chronic stimulation of an immune response by repeated injections appear generally not to be valid except for a very few patients with conditions that require the administration of higher doses of toxin. In some rare instances patients may stop responding to toxin and, in a proportion of these cases, the presence of circulating, toxin neutralising, antibodies can be demonstrated. ${ }^{25}$ Where patients develop antibodies there is no alternative to ceasing treatment, although since the various toxin serotypes do not cross react, the possibility of resuming treatment with another toxin serotype may exist. ${ }^{26}$

The increasing knowledge of the structure and function of the botulinum toxins ${ }^{11,12,14-19}$ offers the possibility of developing new classes of drugs incorporating various biological activities of these toxins. For example, the highly specific binding of the $\mathrm{H}_{\mathrm{c}}$ to the motor end plate may be utilised to target other biologically active molecules to motor nerves. Also, the ability of the $\mathrm{L}_{c}$ component to modulate calciumdependent exocytoses other than acetylcholine release suggests the possibility of controlling other clinical conditions that result from uncontrolled release of mediator substances. It would seem that the remarkable change in our view of the botulinum toxins arising from their initial clinical application may continue with the advent of further, somewhat unexpected, clinical benefits.

P. HAMBLETON

Division of Biologics, PHLS Centre for Applied Microbiology and Research, Porton Down, Salisbury, Wilts SP4 0JG.
8. Shone CC, Hambleton P. Toxigenic clostridia. In: Minton NP, Clarke DJ (eds) Clostridia. New York, Plenum Press. 1989: 265-292.

9. Niemann H. Molecular biology of clostridial neurotoxins. In : Sourcebook of bacterial protein toxins. London, Academic Press. 1991 : 303-348.

10. Harris AJ, Miledi R. The effect of type D botulinum toxin on frog neuromuscular junctions. $J$ Physiol 1971; 217: 497-515.

11. Dreyer F, Mallart A, Brigant JL. Botulinum A toxin and tetanus toxin do not affect presynaptic membrane currents in mammalian motor nerve endings. Brain Res 1983; 270 : 373-375.

12. Dolly JO, Black J, Williams RS, Melling J. Acceptors for botulinum neurotoxin reside on motor nerve terminals and mediate its internalisation. Nature 1984; 307: 457-460.

13. Simpson LC. Peripheral actions of the botulinum toxins. In : Simpson LC (ed) Botulinum neurotoxin and tetanus toxin. New York, Academic Press. 1989: 153-178.

14. de Paiva A, Dolly JO. Light chain of botulinum neurotoxin is 
active in mammalian nerve terminals when delivered via liposomes. FEBS Lett 1990; 277: 171-174.

15. Shone CC, Hambleton P, Melling J. Inactivation of Clostridium botulinum type A neurotoxin by trypsin and purification of two tryptic fragments. Proteolytic action near the $\mathrm{COOH}-$ terminus of the heavy subunit destroys toxin-binding activity. Eur J Biochem 1985; 151 : 75-82.

16. Kozaki S, Ogasawara J, Shimote Y, Kamata Y, Sakaguchi G. Antigenic structure of Clostridium botulinum type B neurotoxin and its interaction with gangliosides, cerebrosides and free fatty acids. Infect Immun 1987; 55: 3051-3056.

17. Shone CC, Hambleton P, Melling J. A 50-kDa fragment from the $\mathrm{NH}_{2}$-terminus of the heavy subunit of Clostridium botulinum type A neurotoxin forms channels in lipid vesicles. Eur J Biochem 1987; 167: 175-180.

18. Schiavo G, Rossetto O, Santucci A, DasGupta BR, Montecucco C. Botulinum neurotoxins are zinc proteins. J Biol Chem 1992; 267: 23479-23483.

19. Schiavo G, Benfenati F, Poulain B et al. Tetanus and botulinum-
B neurotoxins block neurotransmitter release by proteolytic cleavage of synaptobrevin. Nature 1992; 359:832-835.

20. Scott AB. Botulinum toxin injection into extraocular muscles as an alternative to strabismus surgery. Ophthalmology 1980; 87: 1044-1049.

21. Marsden CD, Quinn NP. The dystonias Neurological disorders affecting 20000 people in Britain. BMJ 1990;300: 139-144.

22. Jankovic J, Brin MF. Therapeutic uses of botulinum toxin. New Engl J Med 1991; 324: 1186-1194.

23. Elston JS. Botulinum toxin treatment of blepharospasm. $A d v$ Neurol 1988; 50: 579-581

24. Tsui JKC, Eisen A, Stoessl AJ, Calne S, Calne DB. Doubleblind study of botulinum toxin in spasmodic torticollis. Neurology 1986; 40: 1213-1218.

25. Hambleton P, Cohen HE, Palmer BJ, Melling J. Antitoxins and botulinum toxin treatment. BMJ 1992; 304: 959-960.

26. Ludlow CL, Hallet M, Rhew $\mathrm{K}$ et al. Therapeutic use of type F botulinum toxin. $N$ Engl J Med 1992; 326: 349-350. 